\title{
What competence does a rheumatologist need?: an international perspective
}

\author{
Bernard Amor
}

\begin{abstract}
Objectives-To forecast, on the basis of the past and present position of rheumatologists in Europe, the competence needed in the future to secure and promote the specialty within the healthcare network. Methods-Union Européenne des Médecins Spécialistes/European Board of Rheumatology (UEMS/EBR) questionnaire on $(a)$ training centres in Europe, (b) rheumatologists' skills, (c) UEMS core curriculum.

Results-173 training centres in rheumatology were identified. Reponses to the questionnaire showed both harmony and diversity in the practice of rheumatology. Harmony arises from the need to $(a)$ have an extensive and profound knowledge of, and clinical experience with, all the causes of painful or disabled locomotor apparatus; (b) manage such disorders in the most cost effective way; and (c) promote "shared clinical decision making". The diversity seen both among and within the European countries is due to the different activities of rheumatologists.

Conclusion-Rheumatological competence must be based on a common core, as described in the UEMS core curriculum, and on deeper and diverse clinical or scientific knowledge covering the entire field of the specialty, to secure its flexibility and resilience in the market place and to promote its scientific development. (Ann Rheum Dis 2000;59:580-582)
\end{abstract}

(Union Européenne des Médecins

Spécialistes) and of EBR (European Board of Rheumatology), Rheumatology

Department, Hôpital

Cochin, 27 Fg St

Jacques, 75014 Paris

B Amor

Email: bernard.amor@

cch.ap-hop-paris.fr

Accepted for publication 16 May 2000

The past can help to forecast the future Rheumatology was born as a specialty in many countries around 1960. This new specialty arose from processes having both genetic and environmental origins. The genes of rheumatology came from diverse parent specialties, such as internal medicine, orthopaedics, endocrinology, and spa therapy. These genes are still present in the rheumatology genome. Environ- mental factors have transformed this genetic diversity into a new entity, rheumatology.

Some environmental factors that have stimulated divergence from the parent specialties can be identified:

- Cortisone and non-steroidal antiinflammatory drugs, which have shown that treatment of chronic and disabling diseases is possible. Local steroid treatments, in particular, have stimulated the acquisition of special skills needed for their use

- Rheumatoid factors, which have provided a scientific basis for rheumatic disorders

- The lumbar traction table, which has opened the way to medical treatment of low back pain

- Development of the NHS and social security system, allowing everyone with chronic diseases to have easy access to a healthcare system.

Historical or local factors may have modified the field of rheumatology to a greater or lesser extent in some European countries. A recent survey of rheumatology centres in Europe conducted by the Union Européenne des Médecins Spécialistes/European Board of Rheumatology (UEMS/EBR), such as the French survey, ${ }^{1}$ showed that most of the 186 centres in 12 countries that completed the questionnaire have an interest not only in inflammatory diseases but also in osteoarthritis, metabolic bone diseases, low back pain, and osteoporosis.

The growth of rheumatology has been geometrical from 1960 to 1980 . Since then the curve has become asymptotic. Table 1 gives information on rheumatology training centres in 10 European Union countries.

Rheumatology is not a homogeneous specialty. Some rheumatologists are tertiary care practitioners, whereas others care for local or more diffuse musculoskeletal pain. Occasionally, some provide primary care for patients seen for rheumatological problems. Some are mainly involved in clinical, educational, or basic research programmes.

Table 1 Rheumatology training centres in Europe completing the UEMS/EBR questionnaire in 1999

\begin{tabular}{|c|c|c|c|c|c|c|c|c|c|c|}
\hline & France & $U K$ & Spain & Italy & Netherlands & Switzerland & Portugal & Sweden & Norway & Finland \\
\hline No of centres & 45 & 64 & 32 & 12 & 4 & 18 & 3 & 4 & 3 & 1 \\
\hline Permanent staff* & 138 & 177 & 183 & 81 & 17 & 46 & 22 & 37 & 15 & 3 \\
\hline Attending ${ }^{\star}$ & 319 & 49 & 24 & 26 & 3 & 12 & 0 & 3 & 1 & 0 \\
\hline Trainee ${ }^{\star}$ & 145 & 104 & 106 & 108 & 10 & 61 & 7 & 19 & 10 & 1 \\
\hline Beds* & 1904 & 785 & 241 & 229 & 52 & 868 & 580 & 129 & 62 & 20 \\
\hline Inpatients/year* & 82631 & 19416 & 9071 & 11757 & 1020 & 12686 & & 3443 & 2023 & 700 \\
\hline Outpatients/year ${ }^{\star}$ & 287448 & 462571 & 409017 & 78874 & 38000 & 69637 & & 42390 & 16066 & 6500 \\
\hline
\end{tabular}

${ }^{\star}$ Numbers are the sum of the numbers given by each centre. 
Table 2 Ratio of outpatients to inpatients according to answers to the UEMS/EBR questionnaire on training centres in Europe

\begin{tabular}{lcccc}
\hline Country & Centres (n) & Inpatients & Outpatients & Out/In \\
\hline UK & 64 & 303 & 7227 & 23.8 \\
France & 45 & 1836 & 6387 & 3.4 \\
Italy & 12 & 979 & 6573 & 6.7 \\
Netherlands & 4 & 255 & 9500 & 37.2 \\
Spain & 32 & 756 & 34159 & 45.2 \\
Sweden & 4 & 860 & 10597 & 12.3 \\
Norway & 3 & 674 & 5355 & 7.9 \\
Finland & 1 & 700 & 6500 & 9.2 \\
Switzerland & 18 & 704 & 3868 & 5.5 \\
\hline
\end{tabular}

${ }^{\star}$ Mean by centre.

Differences exist also among academic centres, non-academic hospitals, and private practice. Table 2 illustrates these differences, showing the ratio of outpatients/inpatients in nine European countries that completed the UEMS/ EBR questionnaire on European training centres. The range of this ratio is 3.5 to 45.2 with a mean of 16.8. These differences may be due to differences in the epidemiology of rheumatic diseases, but they are more likely to be due to differences in the field covered by rheumatologists and differences in the healthcare systems.

With time and increasing integration of European countries, these differences tend to disappear. The annual congress of EULAR and the official journal of this society-the Annals of the Rheumatic Diseases - will contribute positively to the expansion of European rheumatology.

\section{What should be happening?}

Genetic and environmental factors, which make rheumatology an active, attractive, and independent specialty, are still operating.

To identify and to forecast the factors which will affect the evolution of a specialty is not easy. Examples from the past and from other branches of medicine are illustrative. On the one hand, who could have imagined with the discovery of streptomycin and isoniazide, major advances in the treatment of chest disease, that a flourishing specialty such as pneumology would be severely affected for many years and finally survive by virtue of its interest in diseases previously neglected, such as chronic bronchitis and lung cancer? On the other hand, centres created to take care of the respiratory distress of poliomyelitis have survived despite the disappearance of this disease because the skill acquired by these centres has been the basis for modern day intensive care units-active and costly departments in many hospitals.

Some changes seen during the past decade have affected or may affect rheumatology in the future. Outpatient care is increasing owing to better knowledge in primary care about rheumatic diseases, easier access to imaging and clinical laboratories, better outcome measurements, and financial pressures. Changes in epidemiology have occurred: rheumatic fever, gout, Reiter's syndrome, bone and joint tuberculosis are less and less common and/or not referred to rheumatology departments in European countries. A trainee might complete his/her training without seeing one of these patients. Increased life expectancy has augmented the incidence of age associated diseases. The complexity and cost of equipment has made some techniques more and more inaccessible to rheumatologists alone (for example, $x$ ray guided injections, biopsies, and arthroscopies), and one of the major issues is the need to delegate or to share some skills with other specialties. The boundaries between primary care, internal medicine, orthopaedics, clinical immunology, and rehabilitation (the parent specialties), but also now pain clinics and unconventional practices, are moving. They are following not only medical interests, scientific and therapeutic innovations but also the healthcare market and healthcare policies, which can modify the distribution of resources in the healthcare system. In practice rheumatology is not doing too badly and has shown its flexibility and resilience in the marketplace.

\section{Can we justify pure specialists in rheumatology?}

For the future, how can our contribution to health care best be described? And why are rheumatologists necessary in the healthcare network?

The long waiting lists for both inpatient and outpatient care in most centres are an indication of patients' confidence in rheumatologists. This clearly shows that people with a wide knowledge and extensive clinical experience of all the reasons that can make the locomotor apparatus painful or disabled, are needed to make a diagnosis rapidly and safely and to treat and manage these disorders in the most cost effective way.

These skills and attitudes are those of a pure specialist, the rheumatologist, because they are poorly taught to others. The painful knee for which an inexperienced doctor asks for a magnetic resonance imaging scan and for which an overly enthusiastic surgeon does an arthroscopy before analysis of the synovial fluid, thus missing the diagnosis of arthritis, is a common story.

Is the future rheumatologist a specialist, a teacher, a scientist, a general practitioner? The goals described above are clearly those of a specialist and of a teacher "who is recognised by the national authority as having completed postgraduate training leading to theoretical knowledge, professional competence, and skills to diagnose, treat, rehabilitate, and prevent rheumatic disorders" (UEMS definition). Efforts to harmonise medical education, regulation of professional qualification, and mutual recognition of diplomas between European countries, in addition to national issues, have been the result of fruitful debates.

Requirements for medical specialist training in rheumatology have been defined according to the UEMS charter on training of medical specialists, and a core curriculum for trainees has been published. ${ }^{2}$ These documents clearly describe, and can help to obtain, the essential core competencies for every rheumatologist, irrespective of country and the type of activity he/she will eventually have. UEMS plans to designate some centres as European Exchange 
Training Centres from among the existing national training centres that wish to acquire this qualification, if the national authority agrees and if the competence for training is confirmed after visiting the centre.

The solutions proposed to obtain the best qualified specialists are not exactly the same for academic and research centres, for inpatient and outpatient departments, and for private practice.

The Calman report on academic rheumatology has been criticised in the United Kingdom because issues concerning the place of research have not been sufficiently taken into account. ${ }^{3}$ In addition, the difficulties in academic rheumatology in America, as recently described, must be prevented. ${ }^{4}$ These difficulties are due to low reimbursement for clinical services, lack of procedures which generate an income, low valuation of cognitive specialists in managed care, and identity diffusion as rheumatologists develop new "product lines" to create "revenue streams", funding difficulties at the NIH, difficulty in attracting and retaining academic rheumatologists, decrease in the number and perceived quality of MD applicants for fellowship, and difficulty in competing with community practices for clinical research.

The academic rheumatologist must be preserved in Europe before this orientation disappears completely by maintaining a good balance of clinician-scholar-educators, and scientifically oriented rheumatologists.

Teaching in outpatient departments, where most of the patients are seen and followed up, must be rethought. ${ }^{5}$ In addition to the essential core competencies, it is desirable to encourage the acquisition of a deeper knowledge of some of the different aspects of rheumatology or of borderline specialties and/or of research, such as epidemiology, pain, sports medicine, physical medicine, genetics, clinical trials, etc. Diversity between rheumatologists is the clue to the resilience and the adaptation of the specialty. Acquisition of this diversity, however, needs to be based on some common requirements. It must be founded on scientific methodology, must contribute to evidence based medicine, and must stimulate observation based medicine

Therefore research should not necessarily be restricted to laboratory studies, particularly for those, who when qualified, will never undertake laboratory research again. That does not mean that basic research will not be encouraged but it is highly probable that in this field, more than in the clinical field, large European or multinational research centres will carry out such research in the future.

Harmonisation does not mean uniformity. Diversity is one of the requirements for species survival. In the coming months many new drugs for the treatment of rheumatology will be proposed, which will be expensive. However, if one of these drugs can cure rheumatoid arthritis it will be a great success, but will also mean a difficult transition for rheumatologists treating this disease only.

Integration of rheumatologists into the healthcare network is an important issue. Rheumatologists must be sufficiently confident in their knowledge and skill to understand, negotiate, and implement treatment within existing or new structures.

Diversity is also important in training centres. This diversity may exist within large centres, but diversity can also be obtained by exchanges nationally or within Europe between the exchange training centres recognised by the UEMS/EBR. We must keep in mind that rheumatology is not a highly technical specialty. Solving clinical problems by a holistic approach and management of chronic diseases are its expertise. To teach and learn this skill, it is necessary to see real patients. Specialists must be confronted with the complex reality. This can be organised through contacts with inpatients or outpatients ${ }^{6}$, and by promoting shared clinical decision making. ${ }^{7}$

\section{Conclusion}

Rheumatologists in Europe must be competent in order to manage the wide spectrum of musculoskeletal conditions that can present to them.

It must be clear to the public, patients, politicians, and other clinicians what skill rheumatologists have to offer, and how patients can be positively helped to improve the quality of their lives.

This aim requires trained rheumatologists in Europe who can share their expertise on an equal footing with other specialists managing musculoskeletal diseases, such as orthopaedists for bone and joint injuries and their sequelae, physical medicine for bone and joint rehabilitation, sports medicine for prevention and treatment of bone and joint injuries and overuse, radiologists for bone and joint imaging, immunologist and physiologists for bone and joint inflammatory diseases physiopathology, pharmacologists, epidemiologists, and statisticians for bone and joint clinical trials. Other specialties also have some connection with rheumatic diseases-namely, dermatology, gastroenterology, and also ophthalmology, cardiology, and, of course, internal medicine itself.

The general practitioner cannot cover the entire field of medicine, and even the skill and knowledge of internal medicine specialists is limited for bone and joint diseases.

Those who can best manage and teach about these wide and diverse diseases, all linked by common anatomical or physiopathological pathways, are rheumatologists, making rheumatology a necessary specialty in the next millennium.

1 Amor B. Survey of the French rheumatology training centres. Rev Rhum Engl Ed 1996;63:493-7

2 Maini RN. The evolution of the core curriculum in the European Board of Rheumatology (UEMS/EBR). Rheumatology in Europe 1998;27:71-5.

3 Isenberg DA, Maini RN. The consequences of the Calman report on academic rheumatology. $\mathrm{Br} \mathrm{J}$ Rheumatol 1996;35:608-10.

4 Pisetsky DS, White B. A college for its teachers. Arthritis Rheum 1999;42:595-8.

5 McDonagh JE. Rheumatology outpatient training: time for a re-think? Ann Rheum Dis 1997;56:701-4

6 Swannell AJ. Specialist training into the new millennium: Swannell AJ. Specialist training into the new millennium:
the price of conformity with Europe. Br J Rheumatol 1996; the price of

7 Carr AJ, Donovan JL. Why doctors and patients disagree. $\mathrm{Br}$ J Rheumatol 1998;37:1-6. 